\title{
Regulatory organization of the staphylococcal sae locus
}

Correspondence

Richard P. Novick

Novick@saturn.med.nyu.edu

Received 2 August 2007

Revised 6 December 2007

Accepted 7 December 2007
Rajan P. Adhikari and Richard P. Novick

The Kimmel Center for Biology and Medicine of the Skirball Institute, and Departments of Microbiology and Medicine, New York University School of Medicine, New York, NY 10016, USA

This paper describes an investigation of the complex internal regulatory circuitry of the staphylococcal sae locus and the impact of modifying this circuitry on the expression of external genes in the sae regulon. The sae locus contains four genes, the sae $R$ and $S$ two-component signalling module (TCS), and sae $P$ and $Q$, two upstream genes of hitherto unknown function. It is expressed from two promoters, $P_{A}$ sae, which transcribes only the TCS, and $P_{C} s a e$, which transcribes the entire locus. A bursa aurealis (bursa) transposon insertion in saeP in a derivative of Staphylococcus aureus NCTC 8325 has a profound effect on sae function. It modifies the activity of the TCS, changing the expression of many genes in the sae regulon, even though transcription of the TCS (from $\mathrm{P}_{\mathrm{A}} \mathrm{sae}$ ) is not interrupted. Moreover, these effects are not due to disruption of saeP since an in-frame deletion in saeP has essentially no phenotype. The phenotype of $S$. aureus strain Newman is remarkably similar to that of the saeP:: bursa and this similarity is explained by an amino acid substitution in the Newman saeS gene that is predicted to modify profoundly the signalling function of the protein. This concurrence suggests that the saeP: : bursa insertion affects the signalling function of saeS, a suggestion that is supported by the ability of an saeQR clone, but not an saeR clone, to complement the effects of the saeP: : bursa insertion.

\section{INTRODUCTION}

sae is a key regulatory locus in staphylococci, coordinating environmental signals with the internal regulatory circuitry governing virulence and other adaptive processes (Novick \& Jiang, 2003). It was identified on the basis of a Tn551 insertion in saeR (Giraudo et al., 1994a), which profoundly affected the expression of a large set of virulence genes (Giraudo et al., 1997), largely, but not entirely, parallel to the regulatory activities of the agr system (Giraudo et al., 1996). The sae locus contains a classical two-component signalling module (TCS), of which SaeS is the receptor kinase and SaeR the response regulator (Giraudo et al., 1999). It also contains two additional genes, saeP and saeQ (Novick \& Jiang, 2003), upstream of the TCS, whose functions are presently unknown. In previous studies (Novick \& Jiang, 2003; Steinhuber et al., 2003), we and others have analysed the transcription/expression pattern of the sae locus, identifying four transcripts, A, B, C and D, and two promoters, $\mathrm{P}_{\mathrm{A}}$ sae and $\mathrm{P}_{\mathrm{C}} s a e$, as diagrammed in Fig. 1(a). Transcript A, $2.1 \mathrm{~kb}$, initiated from promoter $\mathrm{P}_{\mathrm{A}}$ sae and covering saeR and saeS, is present at the earliest time point and diminishes in intensity during the midexponential phase of growth. $\mathrm{P}_{\mathrm{A}}$ sae is within the saeQ

Abbreviation: TCS, two-component signalling module.

A supplementary figure, and tables of primers, are available with the online version of this paper. coding sequence. Transcripts B (2.4 kb), C (3.1 kb) and D $(0.5 \mathrm{~kb})$, all initiated from $\mathrm{P}_{\mathrm{C}}$ sae, appear during the midexponential phase. Transcript $C$ covers the entire locus and may be processed to generate $\mathrm{B}$, which covers $s a e \mathrm{Q}$, saeR and saes; D, which covers saeP only, may be a processed product of $\mathrm{C}$ or it may be a de novo transcript. $\mathrm{P}_{\mathrm{C}}$ sae is (auto)induced by saeR and $S$. Thus, saeR and saeS are transcribed throughout growth, first from promoter $\mathrm{P}_{\mathrm{A}}$ sae and then from $\mathrm{P}_{\mathrm{C}}$ sae. The mid-exponential-phase activation of $\mathrm{P}_{\mathrm{C}}$ sae represents a profound regulatory transition within the sae locus and is critically dependent on various regulatory determinants, including agr, sarA and, as noted, saeR/S, and is affected by various environmental stimuli, including low $\mathrm{pH}$, high $\mathrm{NaCl}$ concentration and subinhibitory concentrations of antibiotics (Novick \& Jiang, 2003; Steinhuber et al., 2003). The present study was initiated with the intention of determining the roles of $s a e P$ and/or $s a e Q$ in the regulatory function of the overall sae locus, starting with a set of bursa aurealis (bursa) insertions, isolated and identified by Bae et al. (2004) and kindly provided by D. Missiakas (University of Chicago), and continuing with an in-frame deletion in saeP. We observed that the bursa insertion and in-frame deletions in $s a e P$ had different effects that could be explained only partially by the polarity of the bursa insertion. In particular, the inframe deletion had no detectable effect on sae transcription or regulatory function, whereas the bursa insertion caused 

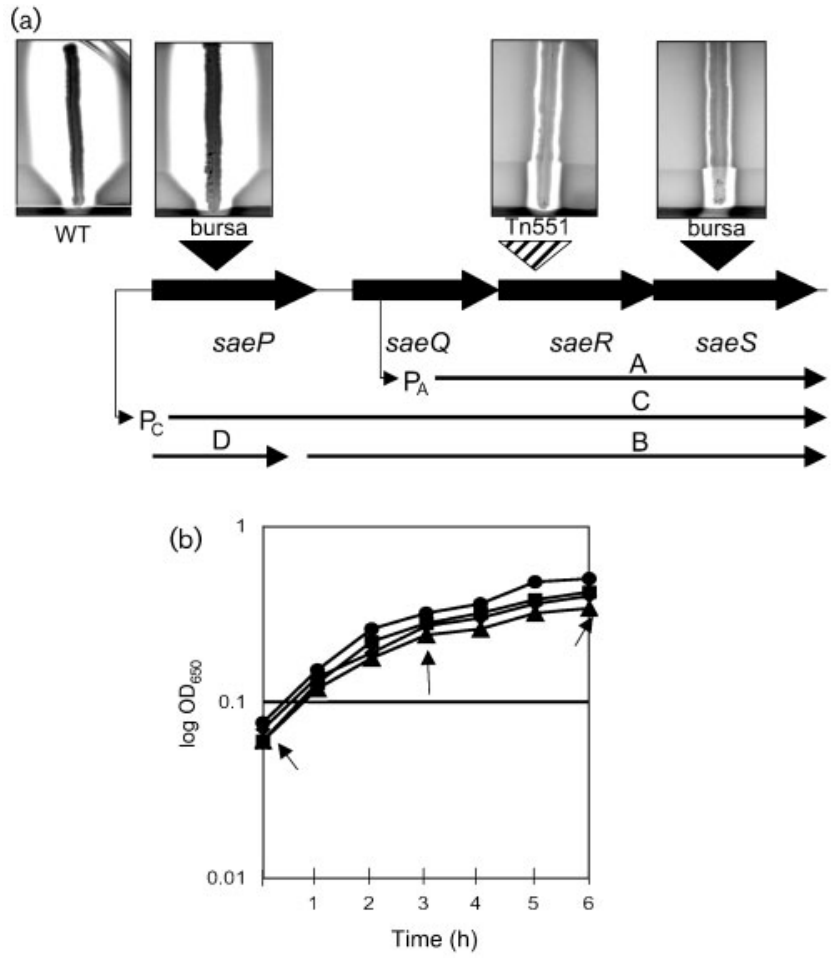

Fig. 1. The sae locus. (a) The sae locus consists of four genes, and is transcribed from two promoters, $P_{A}$ sae and $P_{C} s a e$, generating four transcripts $(A, B, C$ and $D)$ as shown. The two bursa aurealis insertions and the single Tn551 insertion used in this study are indicated by triangles and their haemolytic phenotypes on sheep blood agar are depicted above (see text). (b) Growth curves of saeP $(\bullet), S(\boldsymbol{\bullet})$ and $R(\boldsymbol{\Delta})$ mutants and the WT strain RN6734 ( $\boldsymbol{\square})$. Cultures were grown in side-arm flasks in CYGP without glucose. $t_{0}$ was set at 50 Klett units. The arrows indicate the sampling points $t_{0}, t_{3}$ and $t_{6}$.

a sharp upregulation of $\mathrm{P}_{\mathrm{C}}$ sae, probably owing to subtle effects on downstream components of the sae locus.

The upregulation of $\mathrm{P}_{\mathrm{C}}$ sae by the saeP:: bursa mutation is reminiscent of the sae transcription pattern reported for the wild-type Staphylococcus aureus strain Newman (Blickwede et al., 2005). This led to the rather striking finding that strain Newman, unlike any of nine other $S$. aureus strains for which the sequence of sae is known, has an amino acid substitution in the receptor domain of saeS, which is partially responsible for its phenotype, including the upregulation of $\mathrm{P}_{\mathrm{C}}$ sae and the hyperproduction of coagulase, a phenotype that is rather different from that of NCTC 8325 derivatives and of other S. aureus strains.

\section{METHODS}

Bacterial strains and plasmids. These are listed in Table 1 . In analysing the functions and regulatory circuitry of the sae locus, we have been fortunate in obtaining bursa aurealis insertions (Bae et al., 2004) in all of the genes, kindly provided by Dominique Missiakas
(University of Chicago). We outcrossed all the transposon mutations to several different strains, confirming in all cases linkage of the observed phenotype to the mutation, ruling out adventitious mutations in other genes.

Media and growth conditions. General methods for S. aureus were as described by Novick (1991). Standard CYGP medium without glucose (Novick, 1991) was used for RNA and exoprotein extraction. Culture densities were determined with a Klett-Summerson colorimeter at $540 \mathrm{~nm}$. A Klett reading of 100 corresponds to $\sim 3 \times 10^{8}$ cells $\mathrm{ml}^{-1}$. Bacteria stored at $-80{ }^{\circ} \mathrm{C}$ were inoculated on $\mathrm{GL}$ plates (Novick, 1991) and grown overnight with selective antibiotics as required for plasmid maintenance. For time-course experiments, cultures were resuspended in CYGP to give a Klett reading of 20 $(\mathrm{K}=20)$, grown to $\mathrm{K}=50$ at $37{ }^{\circ} \mathrm{C}$ with shaking, then diluted to $\mathrm{K}=20$ and regrown to $K=50$; this point was taken as time zero $\left(t_{0}\right)$. Samples taken at 3 and $6 \mathrm{~h}$ are listed as $t_{3}$ and $t_{6}$ samples, respectively.

Exoprotein profile and lipase zymogram. For determination of exoprotein profiles, culture samples $(1-10 \mathrm{ml})$ were centrifuged in an Eppendorf centrifuge. The supernatant was recentrifuged to remove any residual organisms, then precipitated with a $10 \%$ volume of $50 \%$ trichloracetic acid, and the pellet analysed by SDS-PAGE according to the method of Laemmli (1970). The lipase zymogram was generated by standard techniques. Briefly, TCA-precipitated exoprotein (native; without reducing agent or boiling) gel was run with TGE buffer. The gel was washed with $20 \%$ 2-propanol and then with water at room temperature. The gel was then transferred onto an agarose plate containing $1 \%$ Tween 20 and incubated overnight at $37{ }^{\circ} \mathrm{C}$. White precipitation represents the lipase activities.

RNA preparation. Cell pellets were treated with RNA Protect reagent (Qiagen) and mechanically disrupted by agitation with glass beads using the Bio101 FastPrep apparatus. RNA was purified using the Qiagen RNeasy kit, and its integrity checked by agarose gel electrophoresis (Novick, 1991).

Northern blot hybridization. DNA samples corresponding to equal numbers of cells were separated by gel electrophoresis through $1 \%$ denaturing agarose (MOPS/formaldehyde), vacuum-blotted to Hybond- $\mathrm{N}^{+}$membranes (Amersham), and UV cross-linked. Blots were hybridized overnight to $\left[\alpha-{ }^{32} \mathrm{P}\right] \mathrm{dATP}-$ labelled, PCR-generated probes. Washed blots were exposed to Phosphorimager screens that were read by a Molecular Dynamics Phosphorimager. Primers (Integrated DNA Technologies) are listed in Supplementary Table S1(a), available with the online version of this paper.

DNA procedures. Most clonings were done by ligating restrictionenzyme-digested PCR products obtained with oligonucleotide primers tagged with restriction sites (Integrated DNA Technologies), as listed in Supplementary Table S1(b), to comparably digested vector DNA.

Primers containing 5' PstI or KpnI sites were used to clone sae genes under the Pcad promoter of pCN51 in pRN9160. Plasmid and chromosomal DNAs were isolated by using a QIAprep Spin Miniprep Kit from Qiagen. PCR products were purified by using a QIAquick PCR Purification kit also from Qiagen. Primers listed in Table S1(b) were used to clone different sae genes and promoter fusions. Primers $7000 \mathrm{PF}$ and $6300 \mathrm{KR}, 6100 \mathrm{PF}$ and $5702 \mathrm{KR}, 5700 \mathrm{PF}$ and $4900 \mathrm{KR}$, $4900 \mathrm{PF}$ and $3900 \mathrm{KR}$ were used to clone saeP, saeQ, saeR and saeS, respectively. Similarly, primers $7000 \mathrm{PF}$ and $3900 \mathrm{KR}, 6100 \mathrm{PF}$ and $3900 \mathrm{KR}, 5700 \mathrm{PF}$ and $3900 \mathrm{KR}, 6100 \mathrm{PF}$ and $4900 \mathrm{KR}$, and $7000 \mathrm{PF}$ and $4900 \mathrm{KR}$ were used to clone saePQRS, saeQRS, saeRS, saeQR and saePQR, respectively. For transcriptional blaZ fusions, primers $7300 \mathrm{PF}$ and $6900 \mathrm{KR}, 6500 \mathrm{PF}$ and $6300 \mathrm{KR}, 6100 \mathrm{PF}$ and $5700 \mathrm{KR}$ were used to clone $\mathrm{P}_{\mathrm{C}}$ sae, $\mathrm{P}_{\mathrm{B}}$ sae and $\mathrm{P}_{\mathrm{A}}$ sae promoters, respectively. Promoters were cloned into pRN7240, upstream of the blaZ reporter 
Table 1. Strains and plasmids

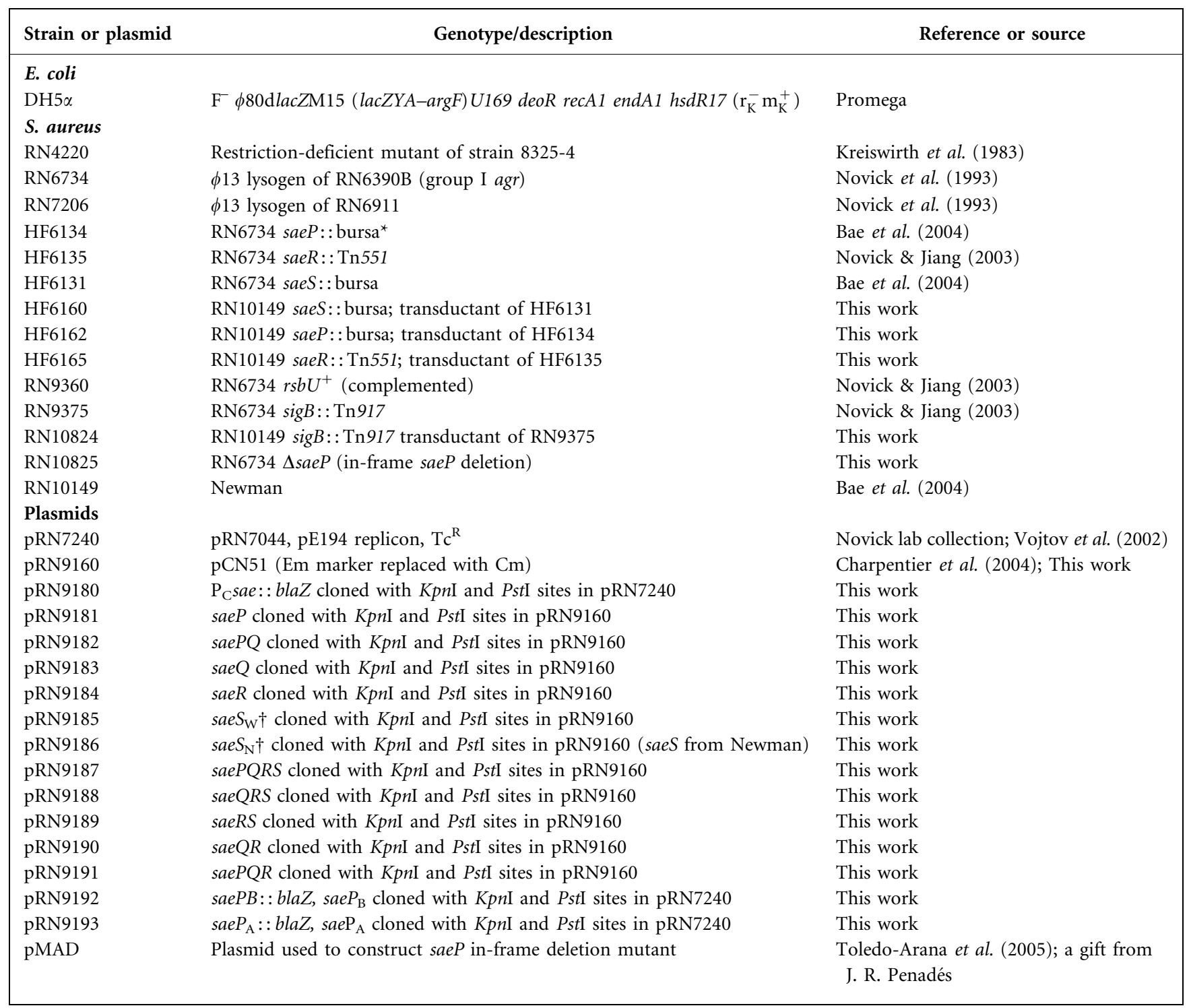

*'bursa' indicates the bursa aurealis transposon.

$\dagger$ sae $S_{\mathrm{W}}$ and $s a e S_{\mathrm{N}}$ are the saeS genes from strains RN6734 and Newman, respectively.

in KpnI and PstI restriction sites. pRN7240 was constructed by replacing the pT181 replicon and $\mathrm{Em}^{\mathrm{R}}$ marker of pRN7044 with the pE194 replicon and tetM marker. pRN9160 contains the cadmiuminducible Pcad promoter, which has a relatively high basal activity and is generally used without cadmium induction (Charpentier et al., 2004; unpublished data). Plasmids were transformed into Escherichia coli $\mathrm{DH} 5 \alpha$ and then moved into S. aureus RN4220 by electroporation (Novick, 1991). All plasmids were first transferred into S. aureus RN4220 then to other $S$. aureus strains by standard transduction techniques with phage $80 \alpha$ (Novick, 1991).

Construction of in-frame saeP mutant. An in-frame saeP deletion was constructed using pMAD (kindly provided by José R. Penadés, Centro de Investigación y Technología Animal, Instituto Valenciano, Spain). We amplified $1 \mathrm{~kb}$ regions at each end of $s a e P$, including four and six amino acid codons at the $\mathrm{N}$ - and C-termini, respectively, using primers listed in Table S1(b). These segments were cloned to pMAD and the resulting construct electroporated into RN4220. Duplications resulting from the Campbell insertion were eliminated by outcrossing and the mutation confirmed by sequencing.

$\boldsymbol{\beta}$-Lactamase assays. To $50 \mu \mathrm{l}$ of sample $10 \mu \mathrm{l} 1 \mu \mathrm{M}$ sodium azide was added. Nitrocefin $(50 \mu \mathrm{l})$ was added as $\beta$-lactamase substrate; activities were recorded using a Thermomax (Molecular Devices) microtitre plate reader. $\beta$-Lactamase units are defined as $\left[V_{\max }\right] / \mathrm{OD}_{650}$ (Ji et al., 1997).

Coagulase test. To $100 \mu \mathrm{l}$ samples, $100 \mu \mathrm{l}$ rabbit plasma (BBL coagulase plasma, rabbit with EDTA) was added and the mixture incubated at $37{ }^{\circ} \mathrm{C}$ for $3 \mathrm{~h}$. Results of twofold dilution series were interpreted as titres, the reciprocal of the highest dilution showing coagulation. 


\section{RESULTS}

Previous studies had suggested that the upstream ORFs, saeP and $Q$, had important but undefined roles in sae regulation (Novick \& Jiang, 2003). To commence the study of their roles, we analysed the effects of available bursa insertions in these two ORFs. As the saeQ insertion was downstream of $\mathrm{P}_{\mathrm{A}} s a e$, its polarity made it uninformative and we have not studied it for this report, concentrating on the saeP::bursa insertion. Although we expected that the saeP transposon insertion would also have polar effects, we found that such effects could not explain all of its properties, and therefore that a comparison of the four congenic strains illustrated in Fig. 1 could provide us with insights into the workings of the locus.

\section{Phenotypic comparison}

On sheep blood agar, the wild-type (WT) strain RN6734 and the saeP::bursa derivative produced high and comparable levels of $\alpha$ - and $\delta$-haemolysins, while the saeR:: Tn551 and saeS: : bursa derivatives produced only $\delta$ haemolysin (Fig. 1a). This shows that the saeP insertion is not significantly polar on $s a e R$ and $S$, confirms that saeR and $S$ are required for $\alpha$-toxin production as previously reported (Giraudo et al., 1994b), and suggests that saeP is not required. For most of the phenotypic comparisons, we sampled at three time points, starting with a culture density of 50 Klett units, namely $t_{0}, t_{3}$ and $t_{6}$. Growth curves of WT and different sae mutants are shown in Fig. 1(b).

To provide a broader comparison of the phenotypes of the four strains, we analysed their exoprotein profiles during the exponential and post-exponential phases of growth (Fig. 2a, b). During the exponential phase, the profiles of WT and saeR and $S$ mutants contained few proteins and looked very similar. A marked increase in the level and number of proteins was observed with the saeP::bursa mutant. One of the proteins induced by the saeP::bursa mutation was identified as coagulase, and a titration assay of coagulase confirmed coagulase production by this mutant but not by the WT or the two other mutants (Table 2). Northern blot hybridization with a coa-specific probe indicated that the observed regulation of $c o a$ is at the level of transcription (Fig. 2c). This was surprising for two reasons: (i) coa, the coagulase gene, has a $\sigma^{\mathrm{B}}$ promoter and $\mathrm{RN} 6734$, a variant of $8325-4$, is $\sigma^{\mathrm{B}}$ defective owing to a deletion in $r s b U$, whose product is required for $\sigma^{\mathrm{B}}$ (a)

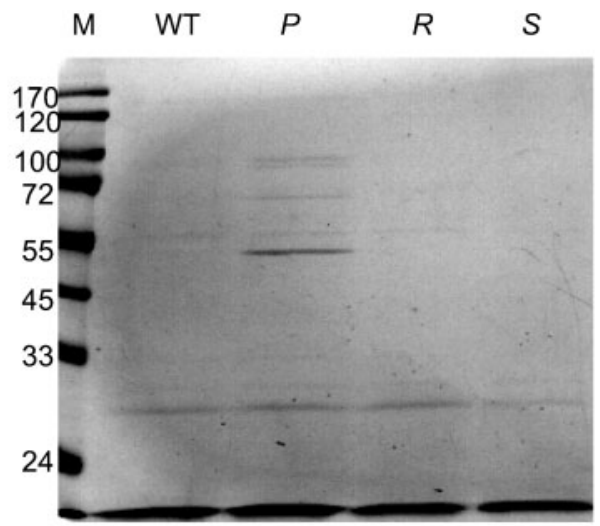

(c) coa

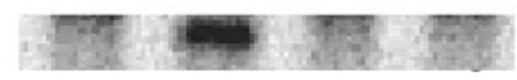

$f n b B$

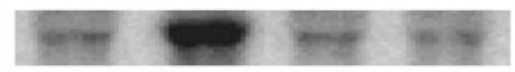

$16 S$

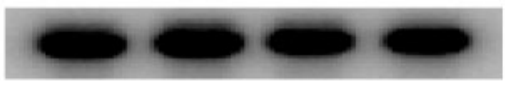

(b)

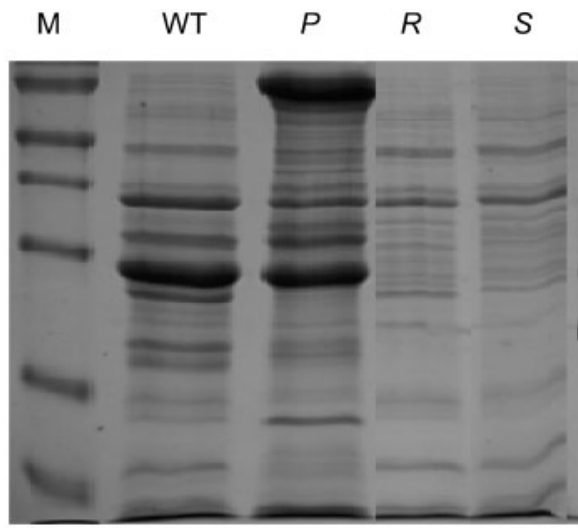

(d)

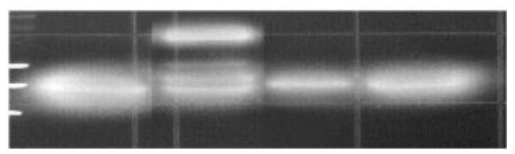

(e)

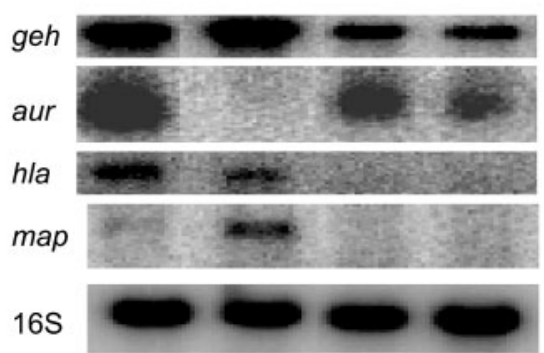

Fig. 2. Effects of transposon insertions in sae genes $P, R$ and $S$. (a) Exoprotein profile at time $t_{0}$ (exponential phase culture at 50 Klett units). (b) Exoprotein profile at time $t_{6}\left(6 \mathrm{~h}\right.$ incubation at $37{ }^{\circ} \mathrm{C}$ with shaking after $\left.t_{0}\right)$. (c) Northern blot at time $t_{0}$ with probes as indicated at left. (d) Tween zymogram (for lipase) at time $t_{6}$. (e) Northern blot at time $t_{6}$. Bacteria were grown in CYGP without glucose. 
Table 2. Coagulase titres

\begin{tabular}{|c|c|c|c|c|c|c|c|c|}
\hline \multirow{2}{*}{ Pcad plasmid } & \multicolumn{5}{|c|}{ RN6734 } & \multicolumn{3}{|c|}{ Newman } \\
\hline & WT & $\Delta s a e P$ & saeP: : bursa & saeR:: Tn551 & saeS : : bursa & WT & saeP: : bursa & saeS : : bursa \\
\hline pRN9160 (vector) & $<1$ & $<1$ & 128 & $<1$ & $<1$ & 256 & 256 & 4 \\
\hline pRN9185 (saeS $\left.{ }_{\mathrm{W}}\right)$ & $<1$ & $<1$ & $<1$ & $<1$ & $<1$ & 8 & $4-8$ & $4-8$ \\
\hline pRN9186 $\left(\right.$ saeS $\left._{\mathrm{N}}\right)$ & & & 128 & & 128 & 256 & 256 & 256 \\
\hline
\end{tabular}

activation (Kullik et al., 1998); (ii) the saeR/S TCS has been reported to upregulate coa (Giraudo et al., 1994a) and these data would suggest that $s a e P$ or a regulatory gene affected by the insertion in saeP downregulates it. Further, the expression of $f n b B$, encoding fibronectin-binding protein $\mathrm{B}$, has also been reported to be dependent on $\sigma^{\mathrm{B}}$ (Bischoff et al., 2004) and $f n b B$ is also expressed in the saeP: : bursa mutant.

The post-exponential-phase exoprotein profiles present a very different picture. The WT and saeP mutant profiles are similar and are characterized by many abundant proteins, whereas the saeR and $S$ mutant profiles are almost identical and are characterized by few, relatively weak bands (Fig. 2b). These results are consistent with upregulation by the TCS of many exoprotein genes (Giraudo et al., 1997) and with the lack of any important effect of saeP: : bursa on the TCS. A significant difference between the WT and saeP mutant profiles is the pronounced pro-Geh (lipase) band in the latter, suggesting that pro-Geh is not processed. A Tween (lipase) zymogram confirmed this (Fig. 2d). Aureolysin is the major pro-Geh processing protease and Northern blot hybridization with an aur (aureolysin gene)-specific probe has shown that aur is not transcribed in the saeP mutant (Fig. 2e), although it is well-transcribed in the WT and the saeR and $S$ mutants. This result suggests that aur is upregulated by saeP and downregulated by saeR and $S$. The Northern blotting data for the three mutants reveal the effects of upstream and downstream mutations and indicate that there is differential regulation of several target genes in addition to aur and geh, including hla (the $\alpha$-haemolysin gene) and map, encoding a secreted adhesin.

\section{sae transcription pattern}

The observed effects of the saeP:: bursa mutation on the transcription of target genes suggested that saeP was involved in the internal regulatory circuitry within the sae locus. Accordingly, we analysed the effect of this insertion on the sae transcription pattern, in comparison with the bursa insertions in saeR and $S$. As noted above, we have previously observed that the RN6734 WT sae locus undergoes a dramatic change in which transcription from the downstream promoter, $\mathrm{P}_{\mathrm{A}}$ sae, generating transcript $\mathrm{A}$, declines and that from the upstream promoter(s), $\mathrm{P}_{\mathrm{C}}$ sae, generating transcripts $\mathrm{B}, \mathrm{C}$ and $\mathrm{D}$, increases sharply in mid-exponential phase (Fig. 3). Transposon insertions had major effects on this pattern. Thus, we observed that a Tn551 insertion in saeR eliminates transcripts B, C and D, and causes constitutive synthesis of an elongated transcript A ( $A^{\star}$ in Fig. 3) (Novick \& Jiang, 2003), suggesting that saeR and $S$ signalling autoinduces $\mathrm{P}_{\mathrm{C}}$ sae and autorepresses $\mathrm{P}_{\mathrm{A}}$ sae. In the present study, we analysed the effects of bursa insertions in saeS and in saeP. In the saeS: : bursa mutant, the upstream transcripts B, C and D were eliminated, as predicted. We were surprised to observe, however, that in the saeP::bursa mutant they were also eliminated. Elimination of $\mathrm{C}$ and $\mathrm{D}$ was expected since the insertion site is within the region specifying these transcripts (Fig. 3). Elimination of B was unexpected: its $5^{\prime}$ end is a considerable distance downstream of the bursa insertion and it has been reported that $\mathrm{B}$ is transcribed from an independent promoter, $\mathrm{P}_{\mathrm{B}}$ sae (Steinhuber et al., 2003). However, a $\beta$ lactamase fusion containing the region expected to include the putative $\mathrm{P}_{\mathrm{B}}$ sae had no activity in any of the strains tested, including WT RN6734 and Newman. Fusions with larger promoter fragments encompassing both $\mathrm{P}_{\mathrm{A}}$ sae and $\mathrm{P}_{\mathrm{B}}$ sae or $\mathrm{P}_{\mathrm{B}}$ sae and $\mathrm{P}_{\mathrm{C}}$ sae had the same activity as the $\mathrm{P}_{\mathrm{A}}$ sae or $\mathrm{P}_{\mathrm{C}}$ sae fusion (not shown). Therefore, it is suggested that $\mathrm{B}$ is a processed form of $\mathrm{C}$ rather than a de novo transcript. In the

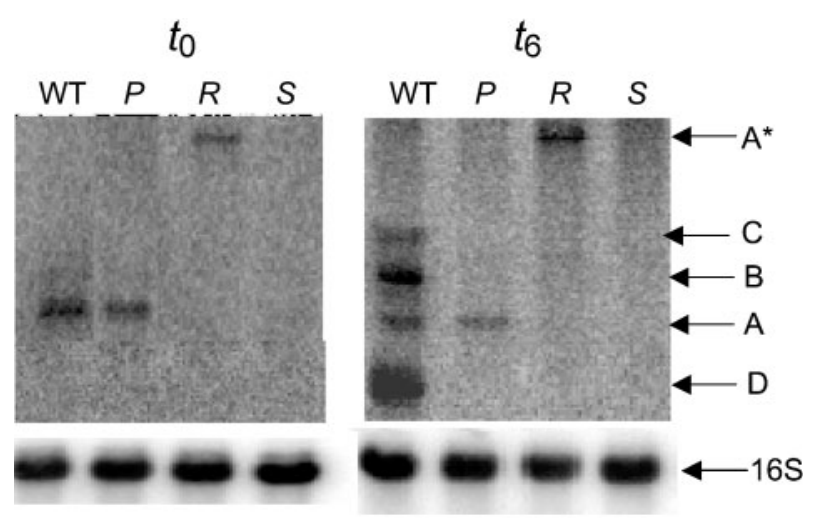

Fig. 3. Northern blot analysis of the sae locus in WT (RN6734) and bursa mutants at times $t_{0}$ and $t_{6}$, with different sae transcripts indicated on the right. A combined probe specific for sae $R$ and saeP was used. $A^{*}$ is the transcript described in our previously published report (Novick \& Jiang, 2003). The primers are listed in Supplementary Table S1(a). 
saeP:: bursa mutant, transcript A was synthesized throughout growth, as in the saeR::Tn551 mutant. These results suggest that the insertion in saeP affects either the level or activation of saeR. They do not, however, explain the difference in target gene expression between the saeP and saeR transposon mutants.

\section{Pcsae promoter activity}

Since saeR and $S$ upregulate $\mathrm{P}_{\mathrm{C}}$ sae (Novick \& Jiang, 2003; Steinhuber et al., 2003), and since the saeP::bursa insertion has a major effect on the sae transcription pattern, it seemed likely that the regulation of $\mathrm{P}_{\mathrm{C}}$ sae would be affected. Accordingly, we constructed and tested a $\mathrm{P}_{\mathrm{C}}$ sae-blaZ fusion. The plasmid containing this fusion was introduced into the WT and saeP, saeR and saeS mutants and $\beta$-lactamase activity was measured as a function of time in a standard growth experiment. As shown in Fig. 4(a), $\mathrm{P}_{\mathrm{C}}$ sae activity in the $\mathrm{WT}$ was low during exponential growth and later increased about sevenfold, while in the saeR and saeS mutants, $\mathrm{P}_{\mathrm{C}}$ sae activity was barely detectable. However, in the saeP mutant, $\mathrm{P}_{\mathrm{C}}$ sae activity was surprisingly high. During early exponential phase, it was eightfold higher than the WT and did not greatly increase beyond this point. The low $\mathrm{P}_{\mathrm{C}}$ sae activity in the saeR and $S$ mutants suggests that the TCS upregulates $\mathrm{P}_{\mathrm{C}}$ sae, and the high $\mathrm{P}_{\mathrm{C}}$ sae activity in the saeP::bursa mutant suggests that saeP or some other regulatory gene whose expression is affected by the insertion downregulates $\mathrm{P}_{\mathrm{C}}$ sae. The mutation thus has a complicated polar effect on the downstream elements of the sae locus; it eliminates readthrough from $\mathrm{P}_{\mathrm{C}}$ sae to $s a e \mathrm{Q}, R$ and $S$, and is predicted to eliminate the post-exponential phase blockage of expression from $\mathrm{P}_{\mathrm{A}}$ sae. (a)

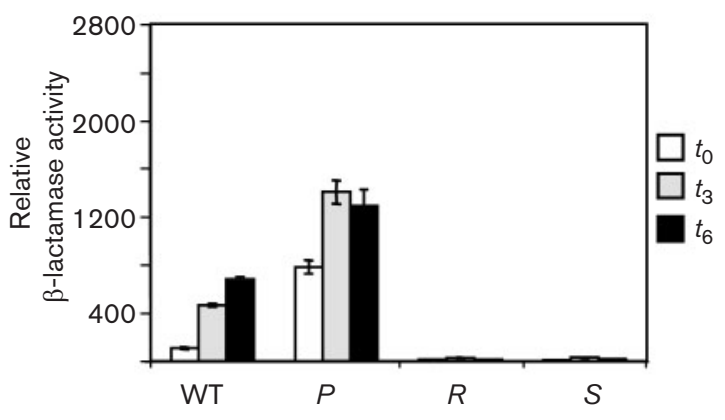

(c) (b)

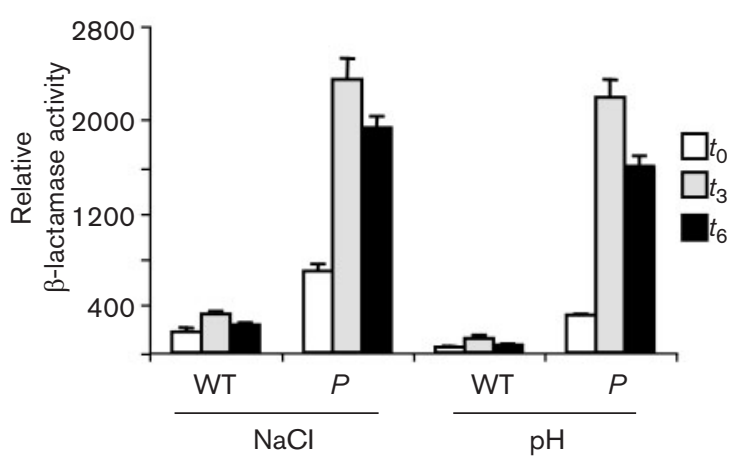

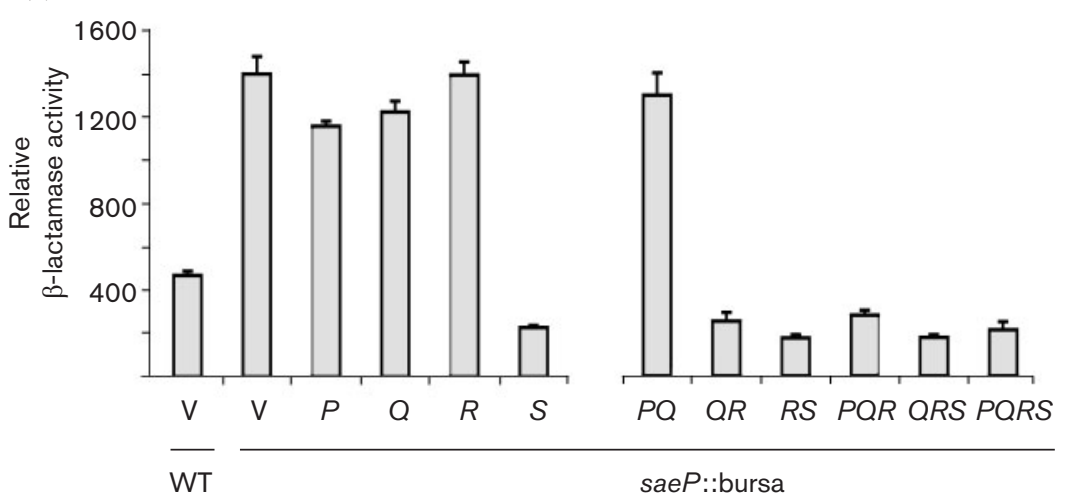

Fig. 4. $\beta$-Lactamase activities of a $P_{C}$ sae-blaZ transcriptional fusion in different strains, over time. WT refers to the wild-type strain (RN6734 in this experiment). $P, R$ and $S$ refer to strains with bursa insertions in sae $P, R$ and $S$, respectively. (a) Timecourse of $\mathrm{P}_{\mathrm{C}}$ sae expression in CYGP at $\mathrm{pH} 7.5$ without salt or glucose added. (b) Effects of $1 \mathrm{M} \mathrm{NaCl}$ and $\mathrm{pH} 5.5$ on $\mathrm{P}_{\mathrm{C}}$ sae expression over time as indicated. The effect of $\mathrm{NaCl}$ was evaluated by incorporating $\mathrm{NaCl}$ at $1 \mathrm{M}$ into CYGP without glucose. The effect of low $\mathrm{pH}$ was evaluated by adjusting the $\mathrm{pH}$ of the medium to 5.5. (c) Complementation of saeP:: bursa by sae subclones. Letters indicate sae gene segments cloned to pCN51 under control of Pcad. Samples were assayed at time $t_{3}$ under standard growth conditions for the $\beta$-lactamase activities of the $\mathrm{P}_{\mathrm{C}}$ sae:: blaZ fusion present in these strains. WT, wild-type strain (RN6734); V, strain containing vector (pRN9160). 
The opposing effects of the upstream and downstream sae mutations were also observed with sae target genes geh and tst (encoding TSST-1).

\section{Effects of the saeP: : bursa mutation on response to environmental factors}

The expression of virulence genes, particularly $t s t$, is exquisitely sensitive to a variety of environmental conditions, including subinhibitory concentrations of antibiotics, high concentrations of $\mathrm{NaCl}$ and low $\mathrm{pH}$ (Chan \& Foster, 1998; Weinrick et al., 2004). Several studies have shown that sae plays an important role in mediating this response (Novick \& Jiang, 2003; Weinrick et al., 2004) and that the $\mathrm{P}_{\mathrm{C}}$ sae activity and, consequently, the postexponential sae transcription pattern are affected, suggesting that sae may sense and respond to these conditions. Previous studies (Novick \& Jiang, 2003; Weinrick et al., 2004) have shown that high salt and low pH block the midexponential activation of $\mathrm{P}_{\mathrm{C}}$ sae and the post-exponential activation of $\mathrm{P} t s t$, raising the possibility that $s a e P$ and/or $s a e Q$, which would not be expressed, transduce these responses. If $\mathrm{SaeP}$ were the transducer, then $\mathrm{P}_{\mathrm{C}}$ sae would no longer be responsive to these signals in strains with the saeP:: bursa insertion. This was, indeed, the case, as shown in Fig. 4(b). In this study, we questioned whether the high activities of $\mathrm{P}_{\mathrm{C}}$ sae in the saeP:: bursa mutant were still sensitive to these environmental signals. As demonstrated with blaZ fusions, the bursa insertion in $s a e P$ eliminated the inhibition by high salt and altered the response to mild acid of the $\mathrm{P}_{\mathrm{C}}$ sae promoter (Fig. $4 \mathrm{~b}$ ).

From all of the above data, it is concluded that the bursa insertion in saeP affects exoprotein production at the transcriptional level, causes a dramatic increase in $\mathrm{P}_{\mathrm{C}}$ sae expression, eliminates the inhibitory effects of high salt and alters the response to low $\mathrm{pH}$.

\section{Effects of an in-frame saeP deletion}

To determine whether inactivation of $s a e P$ is responsible for the observed phenotype of the saeP:: bursa insertion, we constructed an in-frame deletion of saeP. Remarkably, this mutation had no detectable effect on the exoprotein profiles (supplementary Fig. S1A), coagulase activity (Table 2) or Northern blotting patterns for $c o a, f n b B$, geh or aur (not shown). There was also no discernible difference between the sae transcription pattern of the inframe deletion and the WT except that transcripts B, C and D were commensurately shorter (data not shown). The results with the $\mathrm{P}_{C}$ sae-blaZ fusion were consistent with the Northern blotting data. The $\mathrm{P}_{\mathrm{C}}$ sae activity in the mutant and WT were indistinguishable (supplementary Fig. S1B) and the responses to low $\mathrm{pH}$ and high salt were the same in the mutant and WT. The effects of the saeP::bursa mutation are thus dramatically different from those of the $s a e P$ in-frame deletion and suggest that inactivation of $s a e P$ is not responsible for the saeP:: bursa phenotype.
What, then, is responsible for the saeP:: bursa phenotype? This phenotype is clearly distinct from the WT or the other sae mutants analysed.

\section{Complementation tests}

The lack of any effect of the in-frame deletion in saeP suggested that the phenotypic effects of the saeP::bursa mutation would not be complemented by the cloned saeP. As shown in Fig. 4(c), this was confirmed with a plasmid containing saeP under control of the exogenous $\mathrm{P} c a d$. Thus it seemed likely that one or more of the downstream genes are responsible for these effects. Accordingly, we cloned $s a e Q, R$ and $S$ individually behind this same promoter on a multicopy vector. These constructs were introduced into the saeP:: bursa mutant strain, containing also the $\mathrm{P}_{\mathrm{C}}$ saeblaZ fusion, and $\beta$-lactamase activity was measured as a function of time. As shown in Fig. 4(c), Pcad::saeS suppressed the elevated $\mathrm{P}_{\mathrm{C}}$ sae activity of the mutant, restored the response to environmental signals (not shown) and blocked production of coagulase (Table 2). Thus the high level of SaeS fully complemented the saeP:: bursa mutation. As expected, SaeS also complemented the saeS: : bursa mutation (not shown). In other words, the increased expression of saeS, driven by $\mathrm{Pcad}$ and on a multicopy plasmid, downregulated the very high $\mathrm{P}_{\mathrm{C}}$ sae activity of the saeP::bursa mutant and, separately, upregulated the very low $\mathrm{P}_{\mathrm{C}}$ sae activity of the saeS:: bursa mutant. In both cases, $\mathrm{P}_{\mathrm{C}}$ sae activity was restored to the intermediate level characteristic of the WT. These results suggest that either expression of the saeRS signalling module from $\mathrm{P}_{\mathrm{C}}$ sae - such as in the wild-type configuration - or the overexpression of saeS - such as from a high-copy plasmid - may affect sae-responsive genes differently than when the saeRS signalling module is driven by $\mathrm{P}_{\mathrm{A}}$ sae (as with the saeP:: bursa mutant). Alternatively saeS may have different functions depending on its activation state: that is, activated in the WT strain or upon expression from the clone, or not activated in the saeP:: bursa mutant. Since many signal transducers at an increased dosage are activated independently of the usual signal, these possibilities cannot be distinguished on the basis of available data. The fact that saes could not complement the saeR mutant but saeR could (Fig. 5a, d) indicates that $s a e R$, the response regulator, is absolutely required for $\alpha$-haemolysin production, and, as shown in Fig. 4(a), for $\mathrm{P}_{\mathrm{C}}$ sae activity. As expected, the vector $(\mathrm{V})$ alone does not affect the haemolytic pattern of the WT or $S$ and $R$ mutants (Fig. 5b). The fact that saeR could not complement either the saeP or saeS: : bursa mutations suggests that saeR alone, even at an increased dosage, is not activated independently of an activated cognate signal transducer saeS, or that an additional function is required. The fact that neither saeP nor saeQ could complement any of the mutants suggests that neither has a role independent of the TCS.

To analyse the interactions and dependencies of the four sae genes, we constructed additional $\mathrm{P} c a d$ clones with the 
(a)

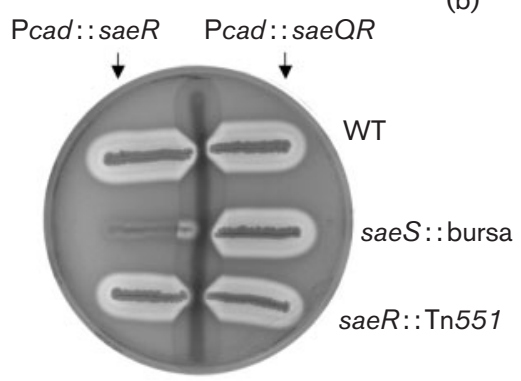

(b)

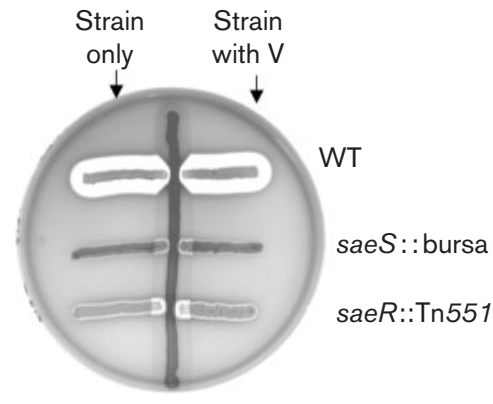

(c)

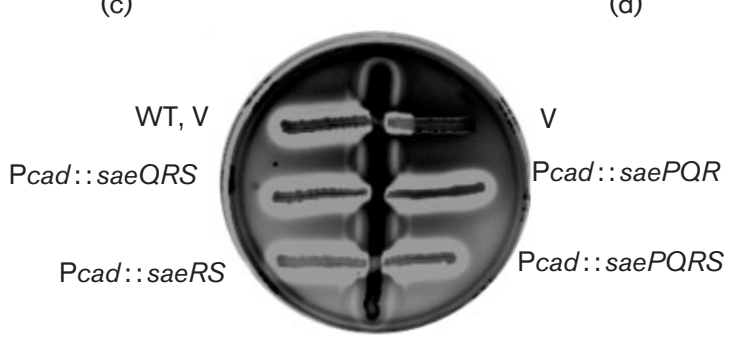

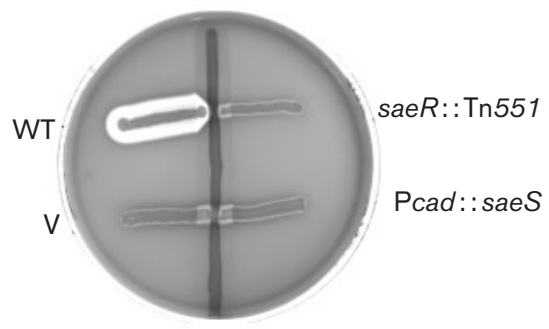

Fig. 5. Haemolytic activities on sheep blood agar. Strains were streaked against RN4220, which produces only $\beta$-haemolysin (turbid zone). (a) Complementation of saeS : : bursa and saeR: :Tn551 by saeR (left) or saeQR (right), cloned to pCN51 behind Pcad. (b) Haemolytic pattern of control strains: WT (RN6734), saeS : : bursa and saeR : :Tn551 (left) or V (vector alone) (right). (c) RN6734-saeR::Tn551 containing the indicated sae subclones, except at upper left, RN6734 (WT) with vector, V (pRN9160). (d) Upper left, WT (RN6734); upper right, saeR: :Tn551; lower left, saeR: :Tn551 with vector (pRN9160); lower right, saeR::Tn551 with plasmid containing Pcad: : saeS. Wide clear zones indicate $\alpha$-haemolysin activity; Narrow clear zones at the intersection with the $\beta$-haemolysin zone indicate $\delta$-haemolysin activity.

sae genes in different combinations. We introduced the plasmids carrying these clones into the different mutant strains harbouring the $\mathrm{P}_{\mathrm{C}}$ sae-blaZ fusion and assayed $\beta$ lactamase activity. As expected, all combinations with saeS, including $S$ alone, $R S, Q R S$ and $P Q R S$, complemented the bursa insertions in both saeP (Fig. 4c) and saes (not shown), and all combinations with $R$, except for $R$ alone, but including $Q R, R S, P Q R, Q R S$ and $P Q R S$, complemented saeP::bursa (Fig. 4c) and saeR::Tn551 (Fig. 5c). However, surprisingly, $Q R$ complemented both saeP and saeS::bursa mutations (Figs $4 \mathrm{c}$ and $5 \mathrm{a}$ ) even though neither gene individually complemented them. A possible explanation for the $s a e Q$ result is that $s a e Q$ on a high-copy plasmid titrates saeR, since $\mathrm{P}_{\mathrm{A}}$ sae is within the $s a e Q$ coding sequence (Fig. 1a) and is repressed by saeR (Novick \& Jiang, 2003). A possible explanation for the saeR result is that saeR evidently does not get activated in the absence of saeS or saeQ. Although saeQ and $R$ are translationally coupled, they do not appear to be required in cis because the saeR clone alone complements the $R$ mutant. In other words, SaeQ has a specific role in sae regulation.

The complementation results suggest that saeQ plays a critical role in sae function, that it acts in conjunction with $s a e R$, that saes is not required when $s a e Q$ and $R$ are expressed from the plasmid and therefore that the role of saeS is either to increase the level of sae $Q$ and $R$ or to activate them. One possible explanation of the effect of the saeP:: bursa mutation is that $s a e R$ and $S$ expressed from $\mathrm{P}_{\mathrm{A}}$ sae are not activated; an alternative possibility is that they are not produced in sufficient amounts to exert their normal regulatory role.

\section{Strain variation}

Not surprisingly, this remarkable and rather subtle regulatory scheme shows variation among $S$. aureus strains, as observed by Blickwede et al. (2005) and by ourselves (unpublished data). For example, the activity of $\mathrm{P}_{\mathrm{C}}$ sae in strain Newman is similar to that seen with the $\operatorname{saeP}:$ : bursa insertion in RN6734, namely a high level of activities (Fig. 6a). One possibility was that the difference was a reflection of $\sigma^{\mathrm{B}}$ activity, which in Newman is typical of most S. aureus strains, but in RN6734 (and all other NCTC 8325 derivatives) is greatly attenuated owing to an inactivating deletion in $r s b U$ (Bischoff et al., 2001). Accordingly, we introduced a $\sigma^{\mathrm{B}}$-inactivating Tn551 insertion into Newman and into an $r s b U^{+}$derivative of RN6734 and compared $\mathrm{P}_{\mathrm{C}}$ sae activity in the resulting $\sigma^{\mathrm{B}}-$ negative strains with that in the respective $\sigma^{\mathrm{B}+}$ strains. Although $\sigma^{\mathrm{B}}$ had a modest stimulatory effect on $\mathrm{P}_{\mathrm{C}}$ sae, only at $t_{0}$, it is clearly not responsible for the difference in $\mathrm{P}_{\mathrm{C}}$ sae activity between the two strains (data not shown). 
(a)

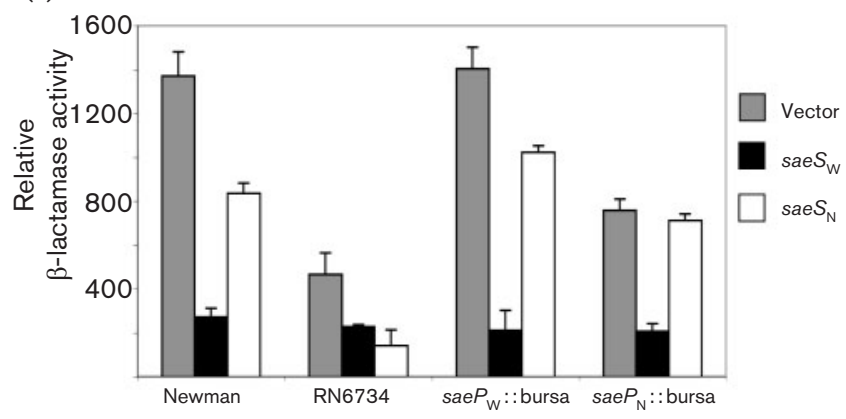

(b)

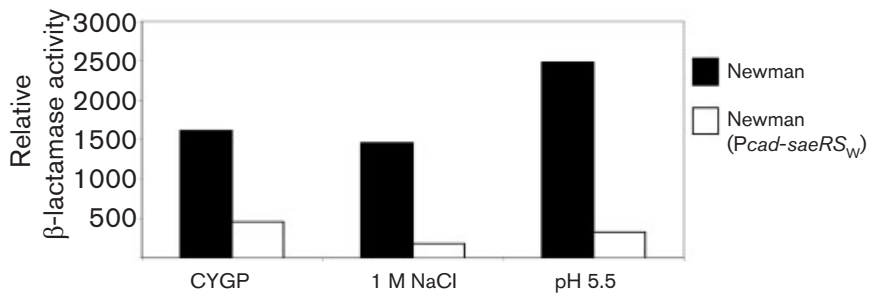

(c)

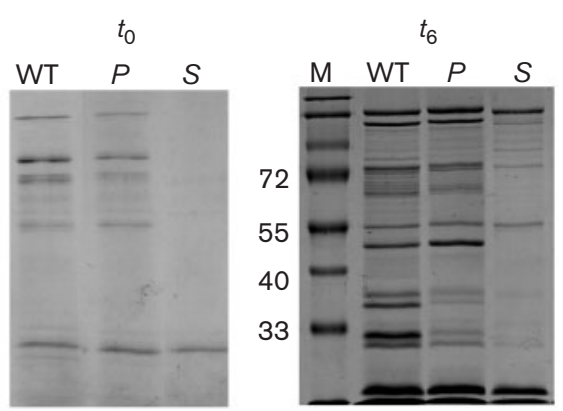

(d)

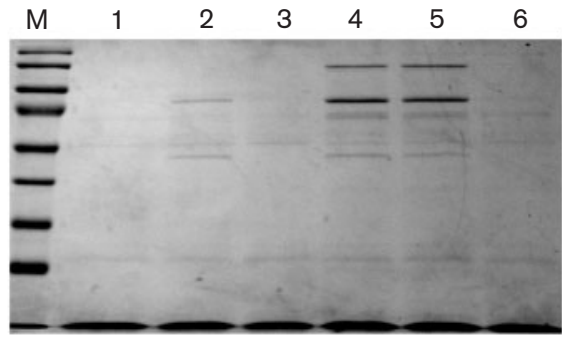

Fig. 6. Functional comparison of saeS genes of strains Newman $\left(s a e S_{N}\right)$ and RN6734 (sae $\left.S_{W}\right)$. (a) $P_{C}$ sae : : blaZ activities in Newman WT, RN6734 WT, RN6734 with the saeP::bursa mutation (sae $P_{\mathrm{W}}:$ :bursa) and Newman with this mutation (sae $P_{\mathrm{N}}:$ : bursa). Strains were assayed with vector alone (grey bars), with cloned sae $\mathrm{S}_{\mathrm{W}}$ (black) or with cloned sae $\mathrm{S}_{\mathrm{N}}($ white). (b) Downregulation of $\mathrm{P}_{\mathrm{C}}$ sae in Newman by cloned RN6734 saeRS (saeRS $S_{\mathrm{W}}$ ): effects of $1 \mathrm{M} \mathrm{NaCl}$ and pH 5.5 are included. (c) Exoprotein profiles of Newman WT and of bursa insertions in Newman saeP or $S$. (d) Cross-complementation of Newman and RN6734 saeP:: bursa by cloned sae $S_{N}$ and cloned sae $S_{W}$, as revealed by exoprotein profiles at time $t_{0}$. Lanes: 1, RN6734; 2, RN6734 saeP: : bursa (cloned sae $S_{N}$ ); 3, RN6734 saeP: : bursa (cloned sae $S_{W}$ ); 4, Newman; 5, Newman (cloned sae $_{\mathrm{N}}$ ); 6, Newman (cloned sae $S_{\mathrm{W}}$ ).

Additionally, the complex sae transcription pattern that develops during growth in RN6734 is fully developed in Newman at $t_{0}$ (not shown), the Newman exoprotein profile at $t_{0}$ is considerably more robust than that of RN6734 (Fig. 6c), and $\mathrm{P}_{\mathrm{C}}$ sae in Newman does not respond to the saeP:: bursa insertion, or to either high salt or low $\mathrm{pH}$ (see below). We have initiated the following study of this difference.

\section{Missense mutation in saes}

We first considered the possibility that there might be a difference between the two sae loci, and found by sequencing that there was a single base substitution in saeS of Newman compared to saeS of RN6734. This base substitution, T53C, resulting in the substitution of proline for leucine at position 18, has recently been confirmed by whole-genome sequencing (Baba et al., 2007). SaeS is predicted to have a single pair of transmembrane segments, in the $\mathrm{N}$-terminal region of the protein, separated by an extracellular segment of 9 amino acid residues; the L18P substitution is within the first of these and is predicted to disrupt the surrounding $\alpha$-helical configuration, changing it to a $\beta$-sheet. This change, within the putative receptor domain, could have a significant effect on the signalling function of the protein. To determine whether this difference was responsible for the difference in sae function in the two strains, we performed a series of crosscomplementation experiments, introducing the cloned saeS from Newman $\left(\right.$ saeS $\left._{\mathrm{N}}\right)$ into an saeS-negative RN6734 and the cloned saeS from RN6734 (saeS $\left.S_{\mathrm{W}}\right)$ into Newman, testing for saeS function by means of the $\mathrm{P}_{\mathrm{C}}$ sae-blaZ fusion, by exoprotein profiling and by coagulase production. As shown in Fig. 6(a, d), the cloned sae $S_{\mathrm{W}}$ converted the functionality of saeS in Newman to that in RN6734, and vice versa, with respect to $\mathrm{P}_{\mathrm{C}}$ sae expression and overall exoprotein profiles. Fig. 6(d) shows that Newman has a fairly robust exoprotein profile at $t_{0}$ (lane 4 ), which is unaffected by the cloned saeS gene from Newman $\left(\operatorname{saeS}_{\mathrm{N}}\right)$ (lane 5), but is converted to the very weak profile seen with RN6734 (lane 1) by the cloned saeS gene from RN6734 $\left(\right.$ sae $\left._{\mathrm{W}}\right)$. Additionally, the saeP:: bursa insertion enhances the exoprotein profile of RN6734 and this is unaffected by cloned saeS $S_{\mathrm{N}}$ (lane 2 - compare with Fig. 2a, lanes 1 and 2) but is reversed by sae $S_{\mathrm{W}}$ (lane 3 ). The $t_{0}$ profiles are shown because these effects are much less apparent at $t_{6}$.

As shown in Fig. 6(b), $\mathrm{P}_{\mathrm{C}}$ sae in Newman did not respond to high salt and was stimulated by $\mathrm{pH} 5.5$, especially at $t_{0}$, in contrast to its behaviour in RN6734 (Fig. 2a); inhibition by both high salt and low $\mathrm{pH}$ was restored in Newman by 
the cloned sae $S_{\mathrm{W}}$. sae $S_{\mathrm{W}}$ also inhibited coagulase production by Newman, whereas sae $S_{\mathrm{N}}$ did not, as shown in Table 2.

\section{DISCUSSION}

Given that sae contains a classical TCS, the normal presumption would be that the response regulator, saeR, is responsible for the regulatory activities of the locus, and this presumption is entirely consistent with the regulatory functions initially described by Giraudo et al. (1999). However, transcription data of the sae locus suggested that the two genes upstream of the TCS, saeP and $Q$, might be involved. In this study, we have begun to examine the functions of these genes. We observed that a bursa transposon insertion in saeP had a striking phenotype, different from the WT or TCS mutants, suggesting that saeP had an independent regulatory role. However, an inframe deletion of saeP had no detectable phenotype.

Two key observations provided a possible interpretation for these strange and seemingly paradoxical results. First, a comparison of NCTC 8325 strains, such as RN6734, with strain Newman revealed that the latter strain had many of the properties described for the saeP:: bursa mutant of RN6734; thus it showed substantial production of coagulase and high activity of $\mathrm{P}_{\mathrm{C}}$ sae, which did not respond to high salt or low $\mathrm{pH}$. These properties of the Newman sae locus were explained by an amino acid substitution in saeS, L18P, in the first transmembrane helix, which is predicted to convert the $\alpha$-helix in this region to a $\beta$-sheet, and which would be expected to have a major effect on the signalling properties of the protein. This substitution, incidentally, is unique to Newman in that it is not present in any of the other genome-sequenced strains. These properties of strain Newman were all converted to the corresponding properties of RN6734 by a plasmid containing the RN6734 saeS gene, whereas a plasmid containing the Newman saeS gene had no effect. Conversely, an RN6734 saeS: :bursa mutant containing the cloned Newman saeS had a phenotype similar to that of the native Newman strain, whereas the plasmid containing the RN6734 saeS gene restored the phenotype of RN6734 saeS: : bursa to that of RN6734. In other words, this finding suggests that the phenotype of the saeP:: bursa insertion in RN6734 might be the result of an effect on saeS signalling. To explain the apparent difference between Newman WT and Newman saeS::bursa, we suggest that SaeS has two different activities depending on its transmembrane signalling function. Our view is that the saeS mutation in Newman does not represent a knockout, but rather locks SaeS in a configuration specific for one of these activities, whereas the bursa insertion simply destroys the gene, eliminating both.

The second clue was provided by a series of complementation experiments (see Figs $4 \mathrm{c}$ and 6 ), in which various sae subclones were tested for their ability to restore the phenotype of the saeP:: bursa mutation, as well as that of other sae mutants to WT. Given that SaeP had no detectable activity, it was not surprising that an saeP clone did not restore the phenotype of the saeP:: bursa insertion mutant to that of the WT. The phenotype was restored, however, by each of several sae subclones containing saeS, supporting the idea that the phenotype of the saeP::bursa insertion represented a deficiency in saeS signalling. Since saeQ is expressed by transcription from $\mathrm{P}_{\mathrm{C}}$ sae but not by transcription from $\mathrm{P}_{\mathrm{A}}$ sae, we tested the cloned saeQ for complementation of saeP:: bursa; although this result was negative, the cloned saeQ and $R$, but not the cloned saeR, restored the phenotype of the saeP:: bursa strain to that of the WT. Although this result suggests that saeQ is necessary, it may not show the expected complementation because it contains $\mathrm{P}_{\mathrm{A}}$ sae, which would be likely to titrate saeR. Further, saeQ does not seem necessary in the presence of high gene dosages of saeR and $S$, as also shown in Fig. 4(c).

We have formulated a working hypothesis based on these observations, which has several features, as follows. (i) SaeR, like other response regulators, exists in two (phosphorylation?) states which have different activities. (ii) SaeQ is required for the conversion (dephosphorylation?) of saeR from one state to the other. (iii) It can accomplish this in the absence of saeS. We note that saeQ is not expressed in the saeP:: bursa mutant, since transcript $\mathrm{C}$ is eliminated and since transcript $\mathrm{A}$ is initiated within the $s a e \mathrm{Q}$ coding sequence. (iv) saeR expressed in the absence of $s a e Q$ in the saeP: : bursa mutant is not converted despite the presence of saeS, and, in this form, induces the expression of $\mathrm{P}_{\mathrm{C}} s a e$, the expression of coa, fnbB, map, etc. (v) the L18P substitution in Newman also prevents the conversion of $s a e R$, and is responsible for the phenotype of Newman and its resemblance to the saeP:: bursa of RN6734.

\section{ACKNOWLEDGEMENTS}

We are grateful to Dunrong Jiang for expert technical assistance. Thanks to Brian Weinrick for plasmid pRN7240. This work was supported by a grant-in-aid from the Skirball Institute to R. P. N.

\section{REFERENCES}

Baba, T., Bae, T., Schneewind, O., Takeuchi, F. \& Hiramatsu, K. (2007). Genome sequence of Staphylococcus aureus strain Newman and comparative analysis of staphylococcal genomes: polymorphism and evolution of two major pathogenicity islands. J Bacteriol 190, 300-310.

Bae, T., Banger, A. K., Wallace, A., Glass, E. M., Aslund, F., Schneewind, O. \& Missiakas, D. M. (2004). Staphylococcus aureus virulence genes identified by bursa aurealis mutagenesis and nematode killing. Proc Natl Acad Sci U S A 101, 12312-12317.

Bischoff, M., Entenza, J. M. \& Giachino, P. (2001). Influence of a functional $\operatorname{sig} B$ operon on the global regulators sar and agr in Staphylococcus aureus. J Bacteriol 183, 5171-5179.

Bischoff, M., Dunman, P., Kormanec, J., Macapagal, D., Murphy, E., Mounts, W., Berger-Bachi, B. \& Projan, S. (2004). Microarray-based 
analysis of the Staphylococcus aureus $\sigma^{\mathrm{B}}$ regulon. J Bacteriol 186, 4085-4099.

Blickwede, M., Goethe, R., Wolz, C., Valentin-Weigand, P. \& Schwarz, S. (2005). Molecular basis of florfenicol-induced increase in adherence of Staphylococcus aureus strain Newman. J Antimicrob Chemother 56, 315-323.

Chan, P. F. \& Foster, S. J. (1998). Role of SarA in virulence determinant production and environmental signal transduction in Staphylococcus aureus. J Bacteriol 180, 6232-6241.

Charpentier, E., Anton, A. I., Barry, P., Alfonso, B., Fang, Y. \& Novick, R. P. (2004). Novel cassette-based shuttle vector system for grampositive bacteria. Appl Environ Microbiol 70, 6076-6085.

Giraudo, A. T., Raspanti, C. G., Calzolari, A. \& Nagel, R. (1994a). Characterization of a Tn551-mutant of Staphylococcus aureus defective in the production of several exoproteins. Can J Microbiol 40, 677-681.

Giraudo, A. T., Raspanti, C. G., Calzolari, A. \& Nagel, R. (1994b). Characterization of a Tn551-mutant of Staphylococcus aureus defective in the production of several exoproteins. Can J Microbiol 40, 677-681.

Giraudo, A. T., Rampone, H., Calzolari, A. \& Nagel, R. (1996). Phenotypic characterization and virulence of a $s a e^{-} a g r^{-}$mutant of Staphylococcus aureus. Can J Microbiol 42, 120-123.

Giraudo, A. T., Cheung, A. L. \& Nagel, R. (1997). The sae locus of Staphylococcus aureus controls exoprotein synthesis at the transcriptional level. Arch Microbiol 168, 53-58.

Giraudo, A. T., Calzolari, A., Cataldi, A. A., Bogni, C. \& Nagel, R. (1999). The sae locus of Staphylococcus aureus encodes a twocomponent regulatory system. FEMS Microbiol Lett 177, 15-22.

Ji, G., Beavis, R. \& Novick, R. P. (1997). Bacterial interference caused by autoinducing peptide variants. Science 276, 2027-2030.

Kreiswirth, B. N., Lofdahl, S., Betley, M. J., O'Reilly, M., Schlievert, P. M., Bergdoll, M. S. \& Novick, R. P. (1983). The toxic shock syndrome exotoxin structural gene is not detectably transmitted by a prophage. Nature 305, 709-712.

Kullik, I., Giachino, P. \& Fuchs, T. (1998). Deletion of the alternative sigma factor $\sigma^{\mathrm{B}}$ in Staphylococcus aureus reveals its function as a global regulator of virulence genes. J Bacteriol 180, 4814-4820.

Laemmli, U. K. (1970). Cleavage of structural proteins during the assembly of the head of bacteriophage T4. Nature 227, 680-685.

Novick, R. P. (1991). Genetic systems in staphylococci. Methods Enzymol 204, 587-636.

Novick, R. P. \& Jiang, D. (2003). The staphylococcal saeRS system coordinates environmental signals with agr quorum sensing. Microbiology 149, 2709-2717.

Novick, R. P., Ross, H. F., Projan, S. J., Kornblum, J., Kreiswirth, B. \& Moghazeh, S. (1993). Synthesis of staphylococcal virulence factors is controlled by a regulatory RNA molecule. EMBO J 12, 3967-3975.

Steinhuber, A., Goerke, C., Bayer, M. G., Doring, G. \& Wolz, C. (2003). Molecular architecture of the regulatory locus sae of Staphylococcus aureus and its impact on expression of virulence factors. J Bacteriol 185, 6278-6286.

Toledo-Arana, A., Merino, N., Vergara-Irigaray, M., Debarbouille, M., Penades, J. R. \& Lasa, I. (2005). Staphylococcus aureus develops an alternative, ica-independent biofilm in the absence of the arlRS twocomponent system. J Bacteriol 187, 5318-5329.

Vojtov, N., Ross, H. F. \& Novick, R. P. (2002). Global repression of exotoxin synthesis by staphylococcal superantigens. Proc Natl Acad Sci U S A 99, 10102-10107.

Weinrick, B., Dunman, P. M., McAleese, F., Murphy, E., Projan, S. J., Fang, Y. \& Novick, R. P. (2004). Effect of mild acid on gene expression in Staphylococcus aureus. J Bacteriol 186, 8407-8423.

Edited by: J. A. Lindsay 